\title{
Transposable elements generate regulatory novelty in a tissue-specific fashion
}

\author{
Marco Trizzino ${ }^{1,2^{*}}$, Aurélie Kapusta ${ }^{3,4}$ and Christopher D. Brown ${ }^{2,5^{*}}$
}

\begin{abstract}
Background: Transposable elements (TE) are an important source of evolutionary novelty in gene regulation. However, the mechanisms by which TEs contribute to gene expression are largely uncharacterized.

Results: Here, we leverage Roadmap and GTEx data to investigate the association of TEs with active and repressed chromatin in 24 tissues. We find 112 human TE families enriched in active regions of the genome across tissues. Short Interspersed Nuclear Elements (SINEs) and DNA transposons are the most frequently enriched classes, while Long Terminal Repeat Retrotransposons (LTRs) are often enriched in a tissue-specific manner. We report across-tissue variability in TE enrichment in active regions. Genes with consistent expression across tissues are less likely to be associated with TE insertions. TE presence in repressed regions similarly follows tissue-specific patterns. Moreover, different TE classes correlate with different repressive marks: LTRs and Long Interspersed Nuclear Elements (LINEs) are overrepresented in regions marked by H3K9me3, while the other TEs are more likely to overlap regions with H3K27me3. Young TEs are typically enriched in repressed regions and depleted in active regions. We detect multiple instances of TEs that are enriched in tissue-specific active regulatory regions. Such TEs contain binding sites for transcription factors that are master regulators for the given tissue. These TEs are enriched in intronic enhancers, and their tissue-specific enrichment correlates with tissue-specific variations in the expression of the nearest genes.

Conclusions: We provide an integrated overview of the contribution of TEs to human gene regulation. Expanding previous analyses, we demonstrate that TEs can potentially contribute to the turnover of regulatory sequences in a tissue-specific fashion.
\end{abstract}

Keywords: Transposons, Gene regulation, Tissue-specific, Transcription factors

\section{Background}

Sequences derived from transposable element (TE) insertions make up roughly half of the length of the human genome. Several TE groups still show transposing activity in humans, including Long Terminal Repeat Retrotransposons (mostly ERV1-LTRs; [1-3]), Long Interspersed Nuclear Elements (LINEs, mostly L1s; [4, 5]), Short Interspersed Nuclear Elements (SINEs) of the Alu families [6, 7], and SINE-VNTR-Alus (SVAs; [8, 9]).

Multiple elegant studies have demonstrated that TE sequences play a functional role in eukaryotic gene regulation [10-32]. Consistently, we recently demonstrated that TEs

\footnotetext{
*Correspondence: marco.trizzino83@gmail.com;

chrbro@pennmedicine.upenn.edu

${ }^{1}$ Gene Expression and Regulation Program, The Wistar Institute, Philadelphia, PA, USA

2Department of Genetics, University of Pennsylvania, Philadelphia, PA, USA Full list of author information is available at the end of the article
}

are the primary source of evolutionary novelty in primate gene regulation, and reported that the large majority of newly evolved human and ape specific liver cis-regulatory elements are derived from TE insertions [33]. Similarly, other studies have shown that the recruitment of novel regulatory networks in the uterus was likely mediated by ancient mammalian TEs [21, 22], and that TEs have a role in pluripotency [34]. Conversely, other researchers have proposed that TE exaptation into regulatory regions is rare [35], and that TE silencing may not be a major driver of regulatory evolution in primates [36].

Given these contrasting lines of evidence, we aimed to shed light on the contribution of TEs to the evolution of the tissue-specific regulation of human gene expression. For this purpose, we took advantage of publicly available data $[37,38]$ to investigate patterns of TE overlap with tissue-specific histone modification states and to characterize the contribution of TEs to tissue-specific 
gene expression. We find that a significant fraction of the existing human TEs are enriched in regions of the genome bearing epigenetic hallmarks of active or repressed chromatin, suggesting they could potentially be actively regulated by the cellular machinery. DNA transposons and SINEs represent the most frequently enriched classes across tissues, while LTR-ERV1s are the TEs that more commonly show tissue-specific enrichment and active regulatory activity. TE enrichment in active and repressed chromatin exhibits tissue-specific patterns. Genes with consistent expression across tissues are less likely to be associated with a local TE insertion. We detect multiple instances of TEs showing tissue-specific enrichment in active and repressed regions, and demonstrate that they contain binding sites for transcription factors that are tissue-specific master regulators.

\section{Results}

\section{Specific TE families are enriched in active and repressed} genomic regions

To investigate the extent to which TEs contribute to the regulation of human gene expression, we leveraged publicly available data from the Roadmap Epigenomics Project [37] and from the GTEx Project [38]. We focused on 24 primary tissues and cell types that were processed by both consortia (Additional file 1: Table S1). Using five different histone modifications (H3K4me1, H3K4me3, H3K36me3, H3K9me3, and H3K27me3), Roadmap segmented the human genome into 15 regulatory classes, reflecting different degrees and types of regulatory activity. We took advantage of this classification to define active (H3K4me1, H3K4me3, H3K36me3) and repressed (H3K9me3, and H3K27me3) chromatin regions in each of the studied tissues.

To test for TE enrichment in active and repressed chromatin, we used the TE-Analysis pipeline ([39]; https://github.com/4ureliek/TEanalysis; Additional file 2). This pipeline is designed to output the TE composition of given features, such as TE counts and TE amounts, aiming to detect potential TE enrichments in the select features. As expected, we find that the majority of human TEs are significantly depleted from regions marked as active by Roadmap histone modifications (mean $83.9 \%$ of TEs; FDR < 5\%; Additional file 3: Table S2). Nevertheless, 112 TE families $(9.07 \%$ of the annotated TE families in the human genome) are significantly enriched in active chromatin in at least one tissue (FDR $<5 \%$; Fig. 1a; Additional file 3: Table S2). These data suggest variability across tissues: aorta, brain anterior caudate, and adipose are the most "permissive" tissues, while right atrium and spleen do not show any significant TE enrichment in active regions (Fig. 1a).

SINEs and "cut and paste" DNA transposons are the classes most frequently enriched in active chromatin (Fig. 1b). SINE families, the most abundant human TEs
(38.8\% of the total), correspond to $43-66 \%$ of the TEs enriched in active regions (FDR $<5 \%$ ), these fractions being more than expected by chance in all tissues (Proportion Test $p<2.2 \times 10^{-16}$ for each tested tissue). Similarly, DNA TEs, that account for $11.3 \%$ of the annotated TEs, represent $29-47 \%$ of the transposons enriched in active regions (Proportion Test $p<2.2 \times 10^{-16}$ for each tested tissue). In general, SINE-Alu elements are the most commonly enriched TEs (Additional file 3: Table S2).

Conversely, LTRs and LINEs are significantly depleted from active genomic regions of all tissues (Proportion Test $p<2.2 \times 10^{-16}$ for each tested tissue; Fig. 1b). Finally, SINE-VNTR-Alus (SVAs), which are the least abundant TEs in the human genome $(0.12 \%$ of the total annotate TEs in the human genome), are significantly overrepresented in active chromatin in 13/24 tissues; Fig. 1b).

We set out to investigate the TEs overlapping active regions. These TEs are depleted in active promoters and intergenic regions, but significantly enriched within active regions inside gene bodies, and in particular in introns (Fisher's Exact Test $p$-values in Fig. 1c). More specifically, 96.3\% of TEs enriched in gene bodies overlap introns, in line with the normally observed distribution of introns and exons in the human genome (Fig. 1c, Fisher's Exact Test $p>0.05$ ). We speculate that genomic regions containing active genes are more frequently accessible, thus providing a substrate for TEs to insert. Moreover, TEs present in the bodies of active genes may be less likely to be silenced than TEs in intergenic regions.

Using the same approach previously described for the active regions, we searched for TEs enriched in repressed genomic regions. Overall, 314 human TE families (25.4\%) are significantly enriched in repressed regions of the genome in at least one tissue (FDR <5\%; Fig. 2a; Additional file 4: Table S3). LTRs (predominantly ERV1) represent the large majority of the repressed TEs (Fig. 2b), followed by LINEs (predominantly L1 s) and DNA TEs. Notably, ERV LTRs and L1 LINEs are among the most active TEs in the genome, and also have their own regulatory architecture $[40,41]$. We thus surmise that these autonomous active TEs may be preferential targets of repressive marks.

We note a very high variability in the number of TE families enriched in repressed regions across tissues (Fig. 2a), as well as large differences in the composition of enriched TE classes in the repressed regions. Notably, the tissues that harbor the highest number of TE families enriched in repressed regions (pancreas, aorta, lung, spleen, esophagus, breast, and liver; Fig. 2a) are also those displaying the highest numbers of enriched LINEs in the same repressed regions (Fig. 2b).

\section{Different TE repression patterns in the human genome} We examined whether TEs preferentially overlap regions repressed via Polycomb Repressive Complex (H3K27me3) 

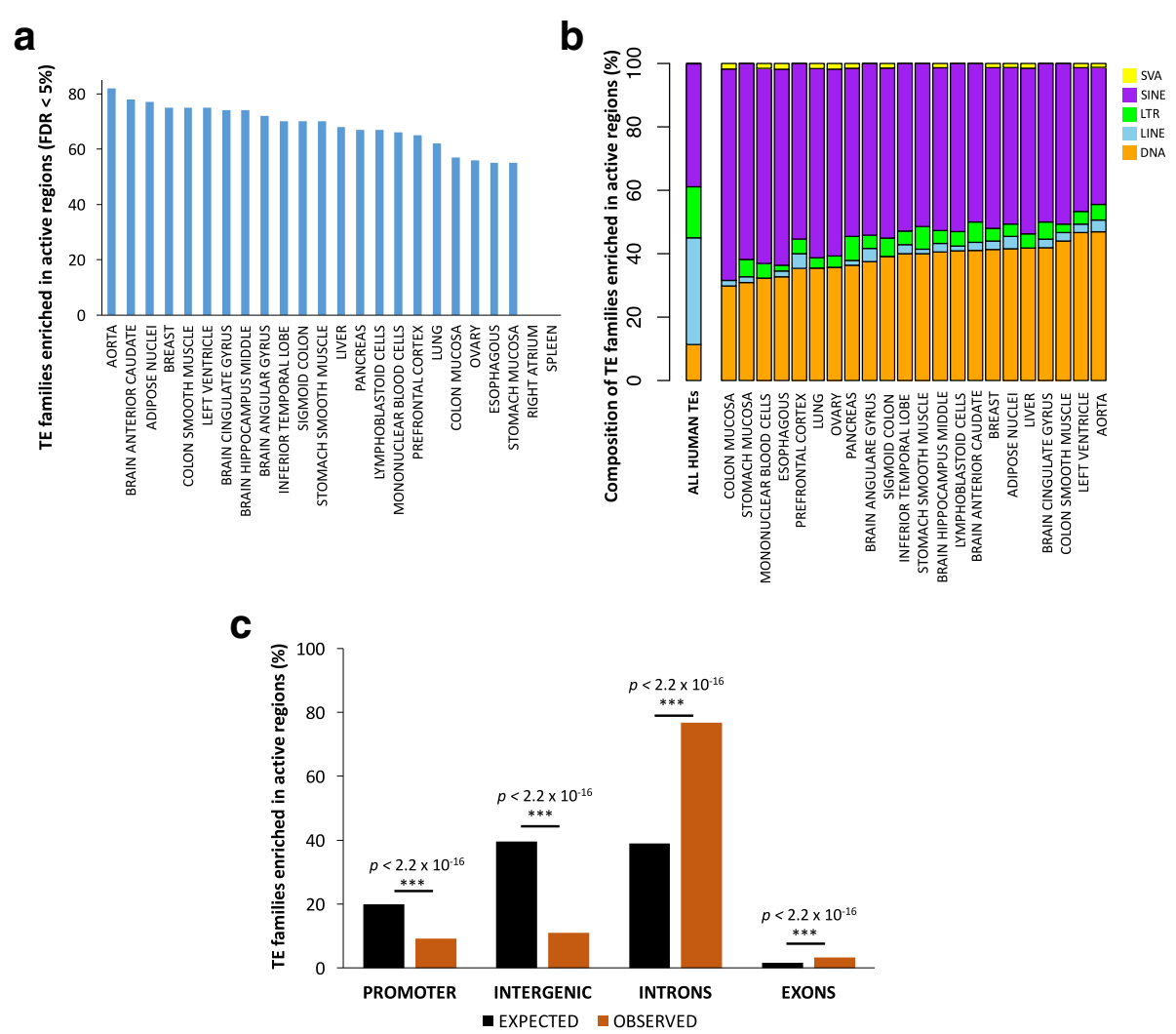

Fig. 1 Transposable elements are enriched in active genomic regions. (a) The plot displays the numbers of enriched TE families in the active genomic regions for each tissue (FDR $<5 \%$ ). The distribution suggests a tissue-specific pattern. (b) Stacked-bar charts show TE class composition for the TE families enriched in active regions (FDR $<5 \%$ ). SINE and DNA transposons are the dominant TEs enriched in active regions. (c) The TEs enriched in active regions are depleted from promoters and intergenic regions, while they are significantly enriched in intronic regions

or via H3K9me3-associated Heterochromatin. Overall, $78.6 \%$ of the regions classified as repressed in the human genome across all tissues are bound by H3K27me3 (Polycomb Repressive Complex), while 21.4\% are marked by H3K9me3 (Heterochromatin conformation). However, when we restrict the analysis to the repressed regions containing a TE, we report an overall higher than expected overlap with H3K27me3 (median across tissues 85.5\%; Proportion Test across tissues $p<2.2 \times 10^{-16}$; Additional file 5: Table S4; Fig. 2C), and a consequent underrepresentation of H3K9me3 (median 15.5\%; Additional file 5: Table S4; Proportion Test $p<2.2 \times 10^{-16}$; Fig. 2d). In $20 / 24$ of the tested tissues, TEs are marked by H3K27me3 more than expected by chance (Proportion Test $p<2.2 \times 10^{-16}$ for each of the 20 significant tissues; Additional file 5: Table S4). In the remaining four tissues, this histone mark is instead underrepresented, while H3K9me3 is overrepresented: breast (H3K27me3 = 76.4\%; Additional file 5: Table S4; Proportion Test $p<2.2 \times 10^{-16}$ ), aorta (55.1\%; Additional file 4: Table S3; $p<2.2 \times 10^{-16}$ ), lung (48.9\%; Additional file 5: Table S4; $p<2.2 \times 10^{-16}$ ), and spleen (26.5\%; Additional file 4: Table S3; $\left.p<2.2 \times 10^{-16}\right)$. Notably, in these four tissues we detect the highest numbers of TE families enriched in repressed regions (Fig. 2a), and the highest proportion of repressed LINEs. We speculate that the heterochromatin state (H3K9me3) may be employed to target specific TE classes and families in a context specific manner [36].

We therefore tested whether different TE classes correlate with either heterochromatin (H3K9me3) or with Polycomb repressed chromatin (H3K27me3). LTRs, LINEs, and SVAs are overrepresented in regions marked by H3K9me3 (Fisher's Exact Test $p<2.2 \times 10^{-16}$; Fig. 2d). Conversely, SINEs and DNA TEs are significantly more likely to overlap H3K27me3 than expected by chance (Fisher's Exact Test $p<2.2 \times 10^{-16}$; Fig. $2 \mathrm{~d}$ ). Notably, SVAs are depleted from the regions marked by H3K27me3 (Fig. 2d).

These findings are consistent with recent reports suggesting that $\mathrm{H} 3 \mathrm{~K} 27 \mathrm{me} 3$ and $\mathrm{H} 3 \mathrm{~K} 9 \mathrm{me} 3$ target different transposon types in embryonic stem cells [42], and with a study reporting that LINEs, LTRs, and SVAs are the most abundant TEs repressed by H3K9me3 in induced pluripotent stem cells [42]. 

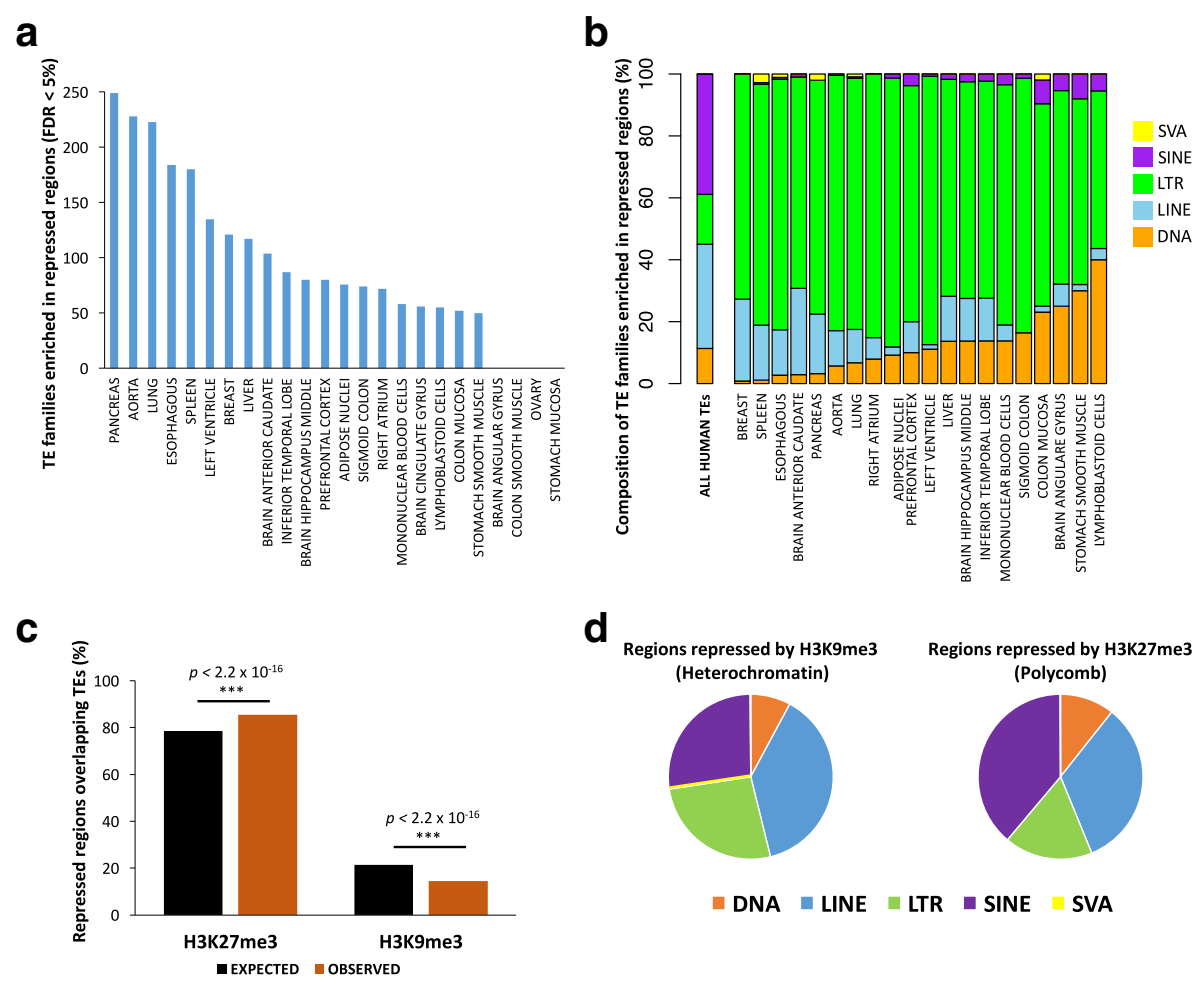

Fig. 2 Transposable elements are enriched in repressed genomic regions. (a) The plot displays the numbers of enriched TE families in the repressed genomic regions for each tissue (FDR $<5 \%$ ). The distribution suggests a tissue-specific pattern. (b) Stacked-chart plot shows class composition for the TE families enriched in repressed regions (FDR < 5\%). (c) Across tissues, the repressed TEs overlap H3K27me3 more than expected by chance, while H3K9me3 is underrepresented. (d) Pie-charts show class composition for the TEs overlapping H3K27me3 and H3K9me3

Ancient TEs are enriched in active regions, while young TEs are repressed

We clustered the annotated human TEs in 35 age classes as in ref. [39] (e.g. Eutheria, Primates, Hominidae; Additional file 6: Table S6), and used the TE-Analysis shuffling script to test for enrichment of each age class in a given set of regions (see Methods). Using this approach, we assessed the age of TEs enriched in active and repressed genomic regions. Ancient TE classes (i.e. age classes older than the Eutheria lineage) are enriched in the active regions of all tested tissues (FDR $<5 \%$; Additional file 6: Table S6). These TEs are largely vertebrate or mammalian specific (Additional file 6: Table S6). Notably, the only tissues with an enrichment of young TEs (specifically primate specific) are blood related (Mononuclear and Lymphoblastoid Cells). These results are in agreement with an elegant study that discovered a key role of primate specific TEs in the regulatory evolution of immune response [25]. TE families enriched in active regions across at least 20 of the 24 tissues correspond to DNA TEs and SINEs (Additional file 3: Table S2). Despite a lack of enrichment of all young TEs taken together in active regions, 24 Alu families are in fact enriched in active regions.
In contrast, young TEs (i.e. TE classes younger than the Eutheria lineage split) are significantly enriched in the repressed regions of most tissues. In particular human specific TEs are enriched in the repressed regions of all brain related tissues (FDR $<5 \%$; Additional file 6 : Table S6). These young TEs correspond to ERV LTRs, L1 LINEs, and SVAs, but only one family is found enriched in at least 20 tissues (MER52A), which is in line with the broad cross-tissue variability of the TEs enriched in repressed chromatin regions (see above).

Collectively, these data suggest that young TEs are predominantly silenced, while the older TE fragments still detectable in the human genome are now more tolerated.

\section{TE insertions are associated with gene expression variance across tissues}

We employed GTEx data to test if TE insertions affect local gene expression. For this purpose, we first assigned each TE overlapping an active genomic region to its nearest gene transcription start site (TSS). Next, we divided all human genes in four categories (Additional file 7: Table S7): 1) Genes associated with TEs that are only found in active regions across tissues; 2) Genes associated with TEs that are 
found in active or repressed regions in a tissue-specific fashion; 3) Genes associated with TEs that are only found in repressed regions; 4) Genes never associated with TE insertions. Based on this classification, genes associated with a TE insertion in regions that are active in at least one tissue are characterized by significantly higher expression variance (normalized by mean expression) than genes either associated to repressed TEs or not associated to a TE (Wilcoxon's Rank Sum Test $p<2.2 \times 10^{-16}$; Fig. 3). Similarly, the genes associated with TEs exclusively found in active regions have significantly higher expression variance than the genes associated with TEs present in both active and repressed regions (Wilcoxon's Rank Sum Test $p=9.91 \times 10^{-}$ ${ }^{8}$; Fig. 3). We reasoned that TE insertions may happen more likely at longer genes located in gene deserts. However, even after correcting our model for gene density and gene length, the gene expression variance is still positively correlated with TE insertion in active regions (linear regression $p<2.2 \times 10^{-16}$ ).

Together, these findings suggest that genes with local TEs overlapping active chromatin have higher variability in gene expression across tissues, and that genes consistently expressed across tissues (e.g. housekeeping and other essential genes) may be less tolerant towards TE insertions in their regulatory regions.

\section{Tissue-specific TE enrichment in active regions correlates} with tissue-specific gene expression

We compared the relative enrichment in active regions of each TE family across tissues. Specifically, for each TE enriched in active regions (FDR $<5 \%$ ), we leveraged the

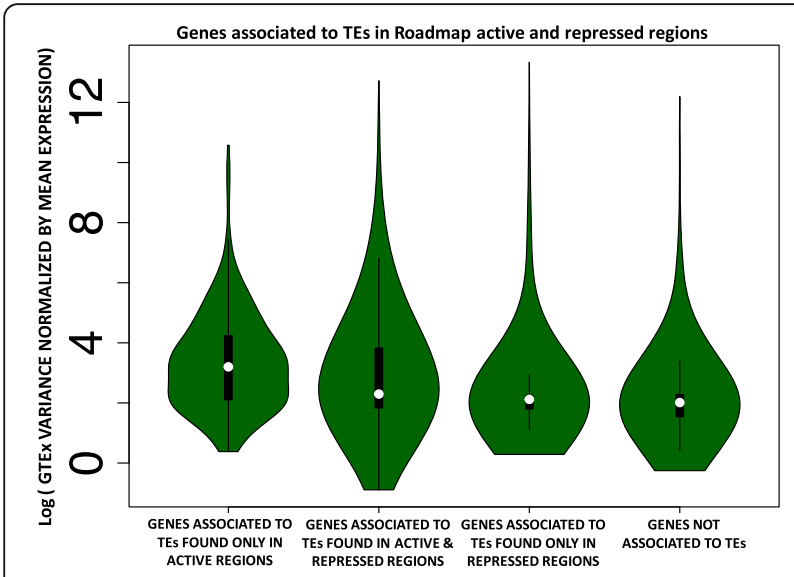

Fig. 3 Genes with higher expression variance are more tolerant towards TE insertion. Human genes were split into four categories: 1) Genes associated with TEs that are only found in active regions across tissues; 2) Genes associated with TEs that are found in active or repressed regions in a tissue-specific fashion; 3) Genes associated with TEs that are only found in repressed regions; 4) Genes never associated with TE insertions. The violin plots display the distribution of the GTEx gene expression variance, normalized by mean expression, for each of the four categories
Odd Ratios from the permutation test of the TE-Analysis pipeline to compute Z-scores (i.e. effect sizes; see methods), and compare them across tissues. We find that TE enrichment varies substantially across tissues (Additional file 8: Table S5; Fig. 4), and many TEs exhibit tissue-specific enrichment in active chromatin (Fig. 4). For example, HERV15 (LTR) is significantly more enriched in the liver and in the stomach mucosa compared to any other tissue (Fig. 4). Motif analysis revealed that the liver regions of active histone modification overlapping HERV15 are enriched in motifs for EOMES (Additional file 9). This transcription factor (TF) has a key role in the hepatic immune response, instructing the development of two distinct natural killer cell lineages specific to this tissue [43]. Moreover, EOMES is also an established tumor suppressor in Hepatocellular Carcinoma [44]. Notably, HERV15 was recovered as significantly enriched in the human liver enhancers also in our previous study [33], suggesting that the findings of the present analysis are not likely to represent batch-specific effects of the Roadmap data.

Similarly, X7C (LINE) and Charlie15a (DNA TE), are the most enriched TEs within regions bearing active chromatin state in the breast. In the sequence of these we find enrichment for binding sites for key breast TFs as KLF5 and CPEB1 (Fig. 5a; Additional file 9). Notably, KLF5 is an essential regulator of hormonal signaling and breast cancer development [45], and is considered a breast cancer suppressor [46]. Similarly, CPEB1 mediates epithelial-to-mesenchyme transition in breast, and mice depleted of this gene showed increased breast cancer metastatic potential [47]. Interestingly Charlie15a shows tissues-specific depletion in the mononuclear blood cells (Fig. 4), highlighting a potential tissue-specific regulatory activity.

To assess the robustness of the enrichment of X7C and Charlie15a in the breast, we ran the TE-Analysis pipeline on publicly available H3K27ac and H3K4me1 data generated by Encode from the breast epithelium and from the MCF7 cell line [48]. Notably, these two TEs were also significantly enriched in the Encode data $(\mathrm{FDR}<5 \%)$, suggesting that batch effects are unlikely strong drivers of this trend.

Analogously, LTR13_ is the most enriched TE in the active chromatin of pancreas and Lymphoblastoid Cell Line (LCL). These LTR copies are enriched for binding sites for SOX9 and PRDM1/Blimp-1 (Fig. 5d; Additional file 9). SOX9 is a master regulator of the pancreatic program [49], while PRDM1/Blimp-1 has a central role in determining and shaping the secretory arm of mature $\mathrm{B}$ Lymphocyte differentiation [50].

We next tested whether tissue-specific TE enrichment in active chromatin (Fig. 4, 5a-f) correlates with tissue-specific-changes in gene expression. Specifically, we tested the TE families showing the highest degree of 


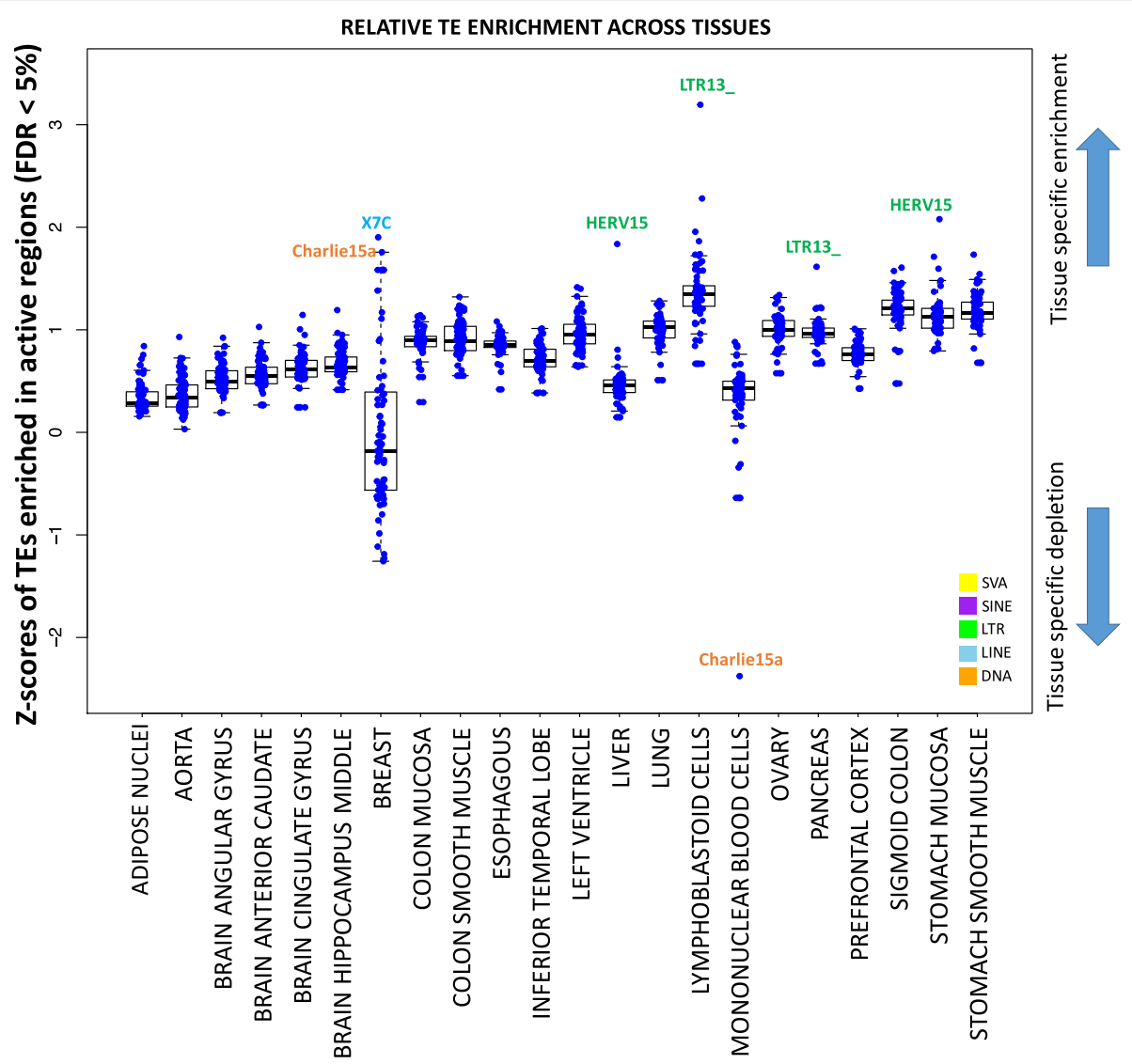

Fig. 4 Transposable elements have tissue-specific enrichment in active regions. The plot displays the distribution of the effect sizes (Z-scores from permutation test, see methods) for each TE enriched in active regions (FDR $<5 \%$ ), in each tissue. The higher the Z-score, the more tissue-specific is the enrichment

tissue-specific enrichment (Fig. 4: HERV15/liver, LTR13_/ LCL, X7C-Charlie15a/breast). With the exception of HERV15/liver (Wilcoxon's Rank Sum Test $p>0.05$ ), in the other tested instances (LTR13_/LCL; X7C-Charlie15a/ breast) the tissue-specific enrichment of the TEs in active chromatin regions is associated with a significant change in the associated gene expression (Wilcoxon's Rank Sum Test $p$-values in Figs. 5b, e). These findings support a possible regulatory role for the co-opted TEs.

To better understand how these tissue-specific TEs may be involved in the regulation of gene expression, we investigated what typology of genomic region they overlap (i.e. promoter, intergenic, introns, exons). Both X7C/ Charlie15a in breast and LTR13_ in LCLs are significantly depleted in promoter and intergenic regions, but overrepresented in gene bodies (Figs. 5c, f), 97.8\% (X7C/ Charlie15a) and 96.4\% (LTR13_) of them respectively found in introns.

The Roadmap data did not include H3K27ac profiles for all tissues. Therefore, to further characterize these intronic regions, we leveraged again the publicly available H3K27ac and H3K4me1 Encode data for the breast
(Breast epithelium and MCF7 cell line; [48]). These data reveal that $57.0 \%$ of the intronic regions containing $\mathrm{X} 7 \mathrm{C}$ or Charlie15a overlap a H3K27ac or H3K4me1 peak, thus suggesting that most of these regions likely represent breast intronic enhancers. As comparison, only $33.7 \%$ of random intronic regions of the same size and number of the ones overlapping X7C/Charlie15a TEs are overlap a H3K27ac or H3K4me1 peak (Fisher's Exact Test $\left.p<2.2 \times 10^{-16}\right)$.

Collectively, these findings point towards a model in which specific TE families, largely belonging to LTR (ERVs) and DNA TE classes, in this context have more regulatory potential than other transposons. Furthermore, our data expand upon previous findings suggesting that ERVs that escape repression can have a significant impact on the host gene regulation $[9,25,26,33,51,52]$.

\section{SVAs exhibit tissue-specific regulatory activity}

In our recent work, we demonstrated that a large fraction of human specific cis-regulatory elements in the liver are SVA transposons, which typically function as transcriptional repressors, at least in this tissue [33]. SVAs are very 


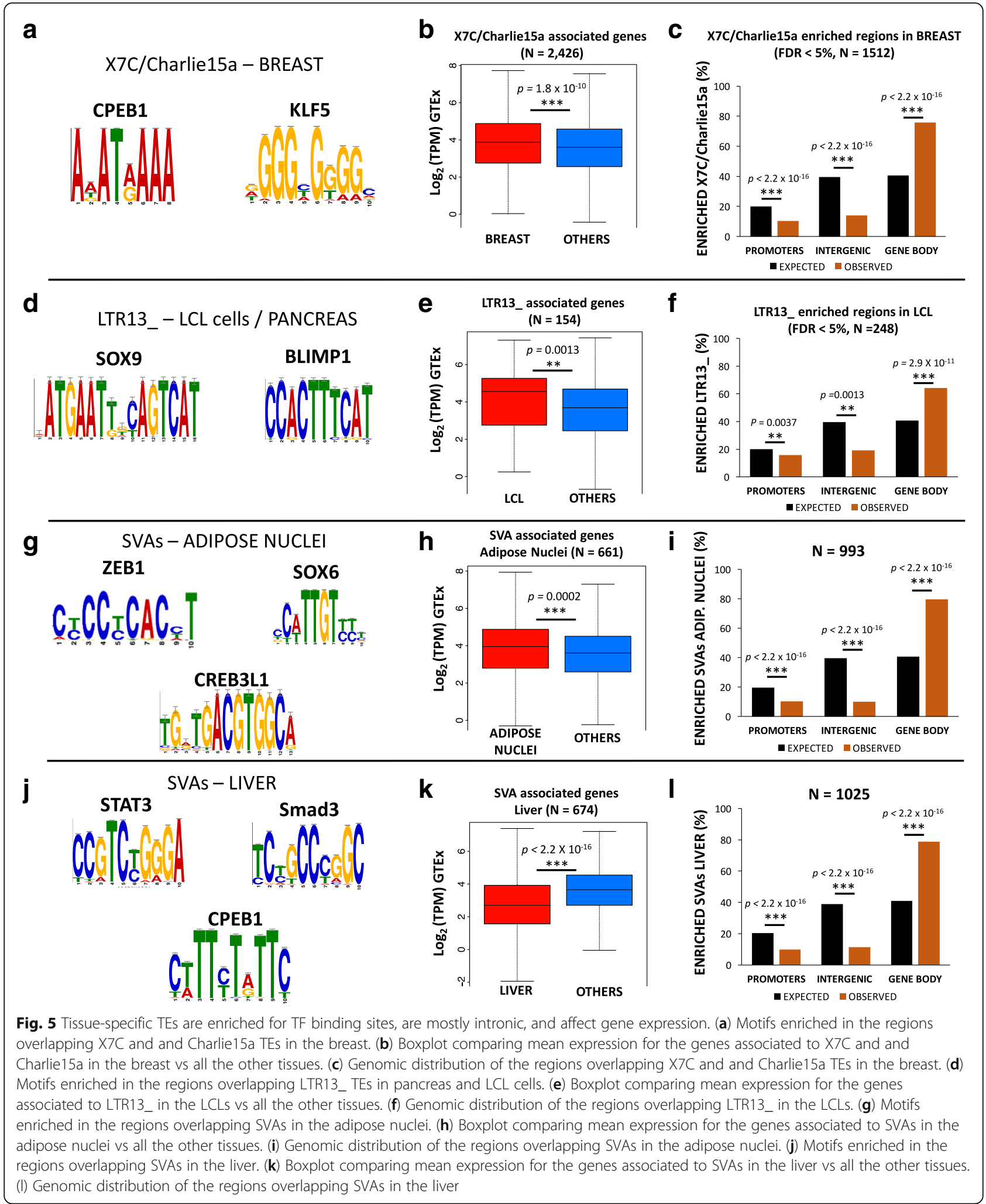


young transposons, being Hominidae (SVA_A, B, C and D) and human specific (SVA_E and F). According to Roadmap data, SVAs are enriched in the active regions of 13/25 tissues (Fig. 1b), and mainly corresponded to SVA_A copies (Additional file 5: Table S4). We first assessed the potential contribution of SVAs to gene regulation of two of these tissues: the adipose nuclei and the liver.

In both tissues, SVAs provide binding sites for key transcription factors (Fig. 5g, j; Additional file 9). ZEB1 is the master regulator of adipogenesis [53, 54], and, based on GTEx data, is ten times more highly expressed in adipose tissue compared to the liver. Similarly, SOX6 contributes to the developmental origin of obesity by promoting adipogenesis, and has a key role in adipocyte differentiation [55]. Consistent with the data reported for other tissues, SVAs associated with active chromatin in adipose nuclei and liver are strongly enriched in gene bodies (Figs. 5i, 1). Genes associated with SVAs in the adipose nuclei are significantly more highly expressed in this tissue compared to other tissues (Wilcoxon's Rank Sum Test $p=0.0002$; Fig. 5h), suggesting that SVA elements can work as transcriptional activators, at least in the adipose tissue.

In the liver, SVAs in active regions are enriched for binding sites of hepatic regulators like CPEB1, that mediates insulin signaling in the liver (Fig. 5j; [56]), and STAT3, that regulates liver regeneration and immune response and negatively modulates insulin action (Fig. 5j; [57]). However, the liver SVAs are also enriched for established transcriptional repressors, like Smad3 (Fig. 5j). Consistently, genes associated with SVAs enriched in active liver regions exhibit lower expression in this tissue compared to all the others (Wilcoxon's Rank Sum Test $p$ $<2.2 \times 10^{-16}$; Fig. 5k), supporting the previously proposed repressive role of SVAs in the hepatic system [33].

\section{Discussion}

The contribution of transposable elements (TEs) to gene regulation was proposed over half a century ago [10-13] and considerably expanded over the last two decades, largely due to the advances in next generation sequencing [14-36].

In order to gain insights in this topic, we identified TEs enriched in active and repressed genomic regions of 24 human tissues, using Roadmap and GTEx data. Our analyses provide a novel integrated overview of the potential impact of TEs to the human gene regulation across multiple tissues, correlating the enrichment of TE copies in active chromatin to tissue-specific gene expression. In fact, many of the previous studies have proposed that TEs are frequently enriched in cis-regulatory elements and lncRNAs [21, 22, 33, 39, 58], but the actual effect of the presence of TEs on the associated gene expression was not tested on a large scale.
Recent work has evaluated the prevalence of TE-derived DNA in enhancers and promoters across mouse cell lines and primary tissues [35]. The present study builds upon this by investigating the dynamics of TE recruitment and the potential effects on tissue-specific gene expression.

We demonstrate that $\sim 10 \%$ of the TEs identified in the human genome are significantly enriched in active regions (promoters, intergenic enhancers, intronic enhancers) of 24 different human tissues. In general, we report a high degree of variability of TE enrichment in the active and repressed genome across tissues, and detect multiple instances of TEs displaying potential tissue-specific regulatory function. We acknowledge that the correlation between tissue-specific TE enrichment in active regions and the tissue-specific changes in gene expression does not necessarily underly a causal role for the TEs. On the other hand, while it is possible that the changes in gene expression are simply due to the presence of a tissue-specific active histone mark, we also find that in all of the tested cases the enriched TE sequence provides binding sites for transcription factors that are master regulators for that specific tissue. This is consistent with the changes in the gene expression of associated (i.e. adjacent) genes and could explain why these TE insertions are retained by selection.

Enriched TEs are typically distributed along gene bodies, likely functioning as intronic enhancers. We reason that this may be explained by the assumption that TEs located within intra-genic regions are less likely to be repressed or removed. In agreement with these findings, a recent study has shown that TEs are depleted in human promoters and intergenic enhancers across multiple tissues [35]. In this context, we see a correlation between gene expression variance and the insertion of TEs in their loci or regulatory regions. This may suggest that genes consistently expressed across tissues are less prone towards TE co-option in their regulatory networks, but future analyses in this direction will be needed to further characterize this phenomenon.

On the other hand, L1 LINEs and ERV LTRs are the most frequently enriched TE classes in the repressed regions. L1 retrotransposons are among the most active TEs in the human genome [59], and several studies have demonstrated that they are also active in brain tissues (e.g. hippocampus), and can contribute to neuronal genetic diversity in mammals [60-63]. Both L1 s and LTRs possess their own regulatory architecture, and we speculate that their preferential silencing prevents these TEs from interfering with gene regulatory networks. Despite this, we demonstrate that LTRs that escape repression may be co-opted in a tissue-specific manner in the active regulatory regions, putatively as a consequence of their regulatory potential. 
We show that TEs enriched in repressed regions of most tissues are generally young, while TEs enriched in active regions of most tissues generally predate the split of eutherian mammals. This is consistent with an accumulation of mutations in these ancient copies that would have increased the likelihood to generate binding sites for transcription factors, and thus the probability for the TE to be co-opted in the regulatory networks. An alternative explanation could be that young TE insertions in active chromatin regions are more likely to be removed by purifying selection than the new insertions in repressed regions, since the latter are more likely to have a neutral impact.

Finally, we demonstrate that SVAs, previously characterized as transcriptional repressors in select cell-types $[33,64]$, can act as both activators or repressors in a tissue-specific fashion.

\section{Conclusions}

In summary, we present a comprehensive overview of the contribution of TE copies to human gene regulation: not only do they provide an important source of evolutionary novelty for the genome, but they can also function with tissue-specific patterns, modulating the expression of key genes and pathways.

\section{Methods}

\section{TE-analysis pipeline}

To test for TE enrichment in active and repressed regions, we used the TE-Analysis pipeline v 4.6 ([39]; https://github.com/4ureliek/TEanalysis). This pipeline is designed to output the TE composition of given features, such as TE counts and TE amounts, aiming to detect potential TE enrichments in the select features. Roadmap annotated BED files (i.e. files listing the coordinates of annotated genomic regions) for each of the 24 tissues were downloaded (http://egg2.wustl.edu/roadmap/data/ byFileType/chromhmmSegmentations/ChmmModels/ coreMarks/jointModel/final/; last access: 10/4/2017). One file per tissue was downloaded ("TISSUE_ID_coreMarks_dense.bed.gz"; Additional file 1: Table S1). From each of the 24 BED files, we produced two different files: one for the regions enriched with epigenomics hallmarks of active chromatin (hereafter "active regions". Histone marks: H3K4me1, H3K36me3, H3K4me3. Roadmap annotations: “TssA”, “TssAFlnk”, “TxFlnk”, “Tx”, “TxWk”, "EnhG”, "Enh", "TssBiv", "EnhBiv"), and one for the regions with signature of repressed chromatin (hereafter: "repressed regions". Histone marks: H3K27me3, H3K9me3. Roadmap annotations: "Het", "ReprPC", "ReprPCWk").

For each tissue, we tested for TE enrichment in the "active" and "repressed" BED files using the "TE-analysis_Shuffle_bed.pl” script v 4.3. Specifically, this script assesses which TEs are significantly enriched in a set of features (BED files) by comparing observed overlaps with the average of $N$ expected overlaps (here 1000). These expected overlaps were obtained by shuffling the genomic position of TEs. TE annotations were downloaded from the University of California Santa Cruz Genome Browser (RepeatMasker, Hg19 version; [65]).

The "TE-analysis_Shuffle_bed.pl" script was run with Bedtools v2.27.1 [66] and the following parameters:

- f Roadmap_BEDFILE (active or repressed)

- q RepeatMasker.out (TE file, hg19)

- n 1000 (number of bootstrap replicates)

- $r$ hg19.chrom.sizes

- g 20141105_hg38_TEage_with-nonTE.txt (distributed with the pipeline)

- s rm. (shuffles the TEs within their genomics position)

The script performs a two-tailed permutation test to assess the enrichment (or depletion) of each annotated $\mathrm{TE}$ in the given regions (Roadmap regions), thus assigning a $p$-value to each annotated TE. Additionally, we corrected for multiple testing by applying a False Discovery Rate (FDR; [67]). Only TEs with FDR $<5 \%$ were retained, considered significantly enriched in the given tissue, and used for downstream analyses.

\section{Composition of enriched TEs}

To characterize TEs enriched within active and repressed regions of each tissue (e.g. Figs. 1b, 2b), each TE was assigned to one of the major TE classes: DNA transposons, LINEs, LTRs, SINEs, SVAs, according to RepeatMasker annotations. To assess the genomic distribution of the enriched TEs (e.g. Figs. 1c, 2c), we considered as 1) PROMOTERS: all of the regions found within $+/-1 \mathrm{~Kb}$ from an annotated TSS (Gencode_v19 comprehensive annotations). 2) GENE BODIES: all of the regions overlapping an annotated gene but not overlapping the promoter region. 3) INTERGENIC: all of the regions not overlapping an annotated gene and distant $>1 \mathrm{~Kb}$ from a TSS.

\section{Correlation between TE insertion and variance in gene expression}

We calculated the variance and mean of the TPM (Transcripts Per Million) for each gene using GTEx data. We assigned each TE overlapping an active or a repressed region to the closest gene, based on the distance to the nearest transcription start site. Next, we divided all human genes in four categories: 1) Genes associated with TEs that are only found in active regions across tissues; 2) Genes associated with TEs that are found in active or repressed regions in a tissue-specific fashion; 3) Genes associated with TEs that are only found in repressed regions; 4) Genes never associated with TE insertions. Gene 
expression variance, normalized by mean expression, was compared across the four categories. Gene density and gene length were used as covariates for the model. Specifically, gene density was calculated as the amount of exonic sequence present within $+/-100 \mathrm{~Kb}$ from each gene. In summary, the following model was used:

$\operatorname{lm}$ (normalized_variance CATEGORY+gene_length + gene_density).

Variance was normalized by average expression across tissues.

\section{Computation of Z-scores for tissue-specificity}

For each TE enriched in active regions $(\mathrm{FDR}<5 \%$ ), we used the Odd Ratios (OR) from the permutation test of the TE-Analysis pipeline to compute Z-scores with the following equation: (OR - mean $(\mathrm{OR}))$ / $\mathrm{sd}(\mathrm{OR})$. Z-scores can be found in Additional file 8: Table S5.

\section{Motif analyses}

Motif analyses were performed using the Meme-Suite [68], and specifically with the Meme-ChIP application. Fasta files of the regions of interest were produced using BEDTools v2.27.1. Shuffled input sequences were used as background. E-values $<0.001$ were used as threshold for significance [68].

\section{Testing for TE co-option on gene expression}

For each human gene and for each tissue, GTEx provides the mean of the TPMs (Transcripts Per Million). To test whether tissue-specific TE enrichment correlates with tissue-specific changes in gene expression, for each gene associated with a TE of interest we used the mean TPMs to compare the expression of genes in the tissue of enrichment Vs the average of the gene expression of the same genes in all the other considered tissues (i.e. mean of TPMs across all the other tissues).

\section{Statistical and genomic analyses}

All statistical analyses were performed using $\mathrm{R}$ v3.4.1 [69]. Figures were made with the package ggplot2 [70]. BEDTools v2.27.1 was used for all the genomic analyses.

\section{Additional files}

Additional file 1: Table S1. Tissues analyzed in the paper, with Roadmap ID codes. List of the 24 tissues analyzed in the paper, with Roadmap ID codes. (XLSX $18 \mathrm{~kb}$ )

Additional file 2: It contains two folders "TE_PIPELINE_SUMMARY_STATISTICS_ACTIVE"

"TE_PIPELINE_SUMMARY_STATISTICS_REPRESSED". In each of the two folders are included multiple spreadsheets (txt format) with the summary statistics of the TE analysis pipeline for ACTIVE and REPRESSED regions respectively. (ZIP $3922 \mathrm{~kb}$ )

Additional file 3: Table S2. $p$-values and corrected $p$-values from TEAnalysis pipeline for active regions (permutation test). For each TE family, uncorrected $p$-values from permutation analysis for ACTIVE regions are reported. Corrected p-values (FDR) for the ENRICHED TEs are found in the second sheet. (XLSX $121 \mathrm{~kb})$

Additional file 4: Table S3. $p$-values and corrected $p$-values from TEAnalysis pipeline for repressed regions (permutation test), For each TE family, uncorrected p-values from permutation analysis for REPRESSED regions are reported. Corrected p-values (FDR) for the ENRICHED TEs are found in the second sheet. (XLSX $188 \mathrm{~kb}$ )

Additional file 5: Table S4. Analysis of histone marks in repressed regions. For each tissue, the numbers of repressed regions overlapping H3K9me3 and H3K27me3 are presented. (CSV $2 \mathrm{~kb}$ )

Additional file 6: Table S6. Analysis of age classes across tissues. FDR values for TE age enrichment/depletion across tissues are presented for the active regions. FDR values for repressed regions are found in the second sheet of the table. (XLSX $27 \mathrm{~kb}$ )

Additional file 7: Table S7. Analysis of correlation between TE insertion and gene expression variance. Normalized GTEx variance for the four gene categories (see Fig. 3), with gene densities and lengths. (TXT $3233 \mathrm{~kb}$ )

Additional file 8: Table S5. Analysis of tissue specific enrichment in active regions. For each TE enriched in active regions (FDR $<5 \%$ ), ZSCORES are presented. (XLSX $38 \mathrm{~kb}$ )

Additional file 9: It contains five "HTML" files with the following names: HERV15_meme-chip.html; LTR13_PANCREAS_meme-chip.html; SVA_ADIPOSE_NUCLEI_meme-chip.html; SVA_LIVER_meme-chip.html; X7C CHarlie15a BREAST ACTIVE.html. The HTML files represent the outputs of the "MEME-ChIP" analyses (i.e. motif analyses) for: HERV15 regions in the liver; LTR13C_ in the pancreas; SVAs in adipose nuclei and liver; X7C_Charlie_15a in Breast. (ZIP 399 kb)

\section{Abbreviations}

FDR: False Discovery Rate; GTEx: Genotype-Tissue Expression Consortium; LCL: Lymphoblastoid Cell Line; LINE: Long Interspersed Nuclear Element; LTR: Long terminal Repeat; OR: Odd Ratio; SINE: Short Interspersed Nuclear Element; SVA: Sine-Vntr-Alu; TE: Transposable Element; TF: Transcription Factor; TSS: Transcription Start Site

\section{Acknowledgements}

We thank Roadmap and GTEx Consortia for the generation of invaluable data. MT thanks his current P.I. (Alessandro Gardini, The Wistar Institute) who granted him time and freedom to work on this project. We also thank the two anonymous reviewers for their valuable suggestions and insights.

\section{Availability of data and materials}

GTEx Expression data (read counts and RPKM; GTEx v6p) are available in the GTEx Portal (http://gtexportal.org). Raw expression data are stored in dbGap (study accession phs000424.v6.p1). However, raw data were not used for the present study, therefore access to dbGap was not necessary.

Complete Roadmap metadata used for the present study are publicly available on GEO: https://www.ncbi.nlm.nih.gov/geo/roadmap/epigenomics/. Roadmap raw data were not used for the present study.

\section{Authors' contributions}

MT and CDB designed the project. MT, AK, and CDB analyzed the data. MT, $A K$, and CDB wrote and approved the manuscript.

Ethics approval and consent to participate

N/A

Competing interests

The authors declare that they have no competing interests.

\section{Publisher's Note}

Springer Nature remains neutral with regard to jurisdictional claims in published maps and institutional affiliations.

\section{Author details}

${ }^{1}$ Gene Expression and Regulation Program, The Wistar Institute, Philadelphia, PA, USA. ${ }^{2}$ Department of Genetics, University of Pennsylvania, Philadelphia, 
PA, USA. ${ }^{3}$ Department of Human Genetics, University of Utah, Salt Lake City, UT, USA. ${ }^{4}$ USTAR, Center for Genetic Discovery, Salt Lake City, UT, USA. ${ }^{5}$ Institute for Biomedical Informatics, University of Pennsylvania, Philadelphia, PA, USA.

\section{Received: 14 May 2018 Accepted: 1 June 2018}

Published online: 18 June 2018

\section{References}

1. Tonjes RR, et al. HERV-K: the biologically most active human endogenous retrovirus family. J Acquir Immune Defic Syndr Hum Retrovirol. 1996;13(1): 261-7.

2. Medstrand P, Mager DL. Human-specific integrations of the HERV-K endogenous retrovirus family. J Virol. 1998;72:9782-7.

3. Fuchs NV, Loewer S, Daley GQ, Izsvak Z, Lower J, Lower R. Human endogenous retrovirus $\mathrm{K}$ (HML-2) RNA and protein expression is a marker for human embryonic and induced pluripotent stem cells. Retrovirology. 2013;10:115

4. Kazazian HH, Wong C, Youssoufian H, Scott AF, Phillips DG, Antonarakis SE. Haemophilia a resulting from de novo insertion of $\mathrm{L} 1$ sequences represents a novel mechanism for mutation in man. Nature. 1988;332:164-6.

5. Brouha B, Schustak J, Badge RM, Lutz-Prigge S, Farley AH, Moran JV, Kazazian HH. Hot L1s account for the bulk of retrotransposition in the human population. Proc Natl Acad Sci U S A. 2003;100:5280-5.

6. Batzer MA, Deininger PL. A human-specific subfamily of Alu sequences. Genomics. 1991:9:481-7.

7. Batzer MA, Gudi VA, Mena JC, Foltz DW, Herrera RJ, Deininger PL. Amplification dynamics of human-specific (HS) Alu family members. Nucleic Acids Res. 1991;19:3619-23.

8. Ostertag EM, Goodier JL, Zhang Y, Kazazian HH. SVA elements are non autonomous retrotransposons that cause disease in humans. Am J Hum Genet. 2003;73:1444-51.

9. Wang H, Xing J, Grover D, Hedges DJ, Han K, Walker JA, Batzer MA. SVA elements: a hominid-specific retroposon family. J Mol Biol. 2005;354:994-1007.

10. McClintock B. The origin and behavior of mutable loci in maize. Proc Natl Acad Sci U S A. 1950;36:344-55.

11. McClintock B. The significance of responses of the genome to challenge. Science. 1984;226:792-801.

12. Britten RJ, Davidson EH. Gene regulation for higher cells: a theory. Science. 1969;165:349-57.

13. Davidson EH, Britten RJ. Regulation of gene expression: possible role of repetitive sequences. Science. 1979;204:1052-9.

14. Jordan IK, Rogozin IB, Glazko GV, Koonin EV. Origin of a substantial fraction of human regulatory sequences from transposable elements. Trends Genet. 2003;19:68-72.

15. Bejerano G, Lowe CB, Ahituv N, King B, Siepel A, Salama SR, Rubin EM, James Kent W, Haussler D. A distal enhancer and an ultraconserved exon are derived from a novel retroposon. Nature. 2006;441:87-90.

16. Wang T, Zeng J, Lowe CB, Sellers RG, Salama SR, Yang M, Burgess SM, Brachmann RK, Haussler D. Species-specific endogenous retroviruses shape the transcriptional network of the human tumor suppressor protein p53. Proc Natl Acad Sci U S A. 2007:104:18613-8.

17. Bourque $G$, Leong $B$, Vega VB, Chen $X$, Lee $Y L$, Srinivasan KG, Chew J-L, Ruan Y, Wei C-L, Ng HH, et al. Evolution of the mammalian transcription factor binding repertoire via transposable elements. Genome Res. 2008;18: 1752-62.

18. Sasaki T, Nishihara H, Hirakawa M, Fujimura K, Tanaka M, Kokubo N, KimuraYoshida C, Matsuo I, Sumiyama K, Saitou N, et al. Possible involvement of SINEs in mammalian-specific brain formation. Proc Natl Acad Sci U S A. 2008;105:4220-5.

19. Markljung $E$, Jiang $L$, Jaffe JD, Mikkelsen TS, Wallerman $O$, Larhammar $M$, Zhang X, Wang L, Saenz-Vash V, Gnirke A, et al. ZBED6, a novel transcription factor derived from a domesticated DNA transposon regulates IGF2 expression and muscle growth. PLoS Biol. 2009;7:e1000256.

20. Kunarso G, Chia NY, Jeyakani J, Hwang C, Lu X, Chan YS, Ng HH, Bourque G. Transposable elements have rewired the core regulatory network of human embryonic stem cells. Nat Genet. 2010;42:631-4.

21. Lynch VJ, Leclerc RD, May G, Wagner GP. Transposon-mediated rewiring of gene regulatory networks contributed to the evolution of pregnancy in mammals. Nat Genet. 2011;43:1154-9.
22. Lynch VJ, Nnamani MC, Kapusta A, Brayer K, Plaza SL, Mazur EC, Emera D, Sheikh SZ, Grützner F, Bauersachs S, et al. Ancient transposable elements transformed the uterine regulatory landscape and transcriptome during the evolution of mammalian pregnancy. Cell Rep. 2015;10:551-61.

23. Schmidt D, Schwalie PC, Wilson MD, Ballester B, Gonçalves Â, Kutter C, Brown GD, Marshall A, Flicek P, Odom DT. Waves of retrotransposon expansion remodel genome organization and CTCF binding in multiple mammalian lineages. Cell. 2012;148:335-48.

24. Chuong EB, Rumi MAK, Soares MJ, Baker JC. Endogenous retroviruses function as species-specific enhancer elements in the placenta. Nat Genet. 2013;45:325-9.

25. Chuong EB, Elde NC, Feschotte C. Regulatory evolution of innate immunity through co-option of endogenous retroviruses. Science. 2016;351:1083-7.

26. Jacques PE, Jeyakani J, Bourque G. The majority of primate-specific regulatory sequences are derived from transposable elements. PLoS Genet. 2013;9:e1003504.

27. Xie M, Hong C, Zhang B, Lowdon RF, Xing X, Li D, Zhou X, Lee HJ, Maire CL, Ligon KL, et al. DNA hypomethylation within specific transposable element families associates with tissue-specific enhancer landscape. Nat Genet. 2013; 45:836-41.

28. del Rosario RCH, Rayan NA, Prabhakar S. Noncoding origins of anthropoid traits and a new null model of transposon functionalization. Genome Res. 2014;24:1469-84.

29. Sundaram V, Cheng Y, Ma Z, Li D, Xing X, Edge P, Snyder MP, Wang T. Widespread contribution of transposable elements to the innovation of gene regulatory networks. Genome Res. 2014;24:1963-76.

30. Pavlicev M, Hiratsuka K, Swaggart KA, Dunn C, and Muglia L Detecting endogenous retrovirus-driven tissue-specific gene transcription. Genome Biol Evol. 2015;7(4):1082-97.

31. Du J, Leung A, Trac C, Lee M, Parks BW, Lusis AJ, Natarajan R, Schones DE. Chromatin variation associated with liver metabolism is mediated by transposable elements. Epigenetics Chromatin. 2016;9:28.

32. Rayan NA, Del Rosario RCH, Prabhakar S. Massive contribution of transposable elements to mammalian regulatory sequences. Semin Cell Dev Biol. 2016;57:51-6.

33. Trizzino M, Park S, Holsbach-Beltrame M, Aracena K, Mika K, Caliskan M, Perry GH, Lynch V, Brown CD. Transposable elements are the primary source in the primate gene regulation. Genome Res. 2017;27: 1623-33.

34. Macfarlan TS, et al. Embryonic stem cell potency fluctuates with endogenous retrovirus activity. Nature. 2012;487:57-63.

35. Simonti CN, Pavlicev M, Capra JA. Transposable element exaptation into regulatory regions is rare, influenced by evolutionary age, and subject to pleiotropic constraints. Mol Biol Evol. 2017;34(11):2856-2869.

36. Ward M, Zhao S, Luo K, Pavlovic B, Karimi MM, Stephens M, Gilad Y. Silencing of transposable elements may not be a major driver of regulatory evolution in primate induced pluripotent stem cells. eLife. 2018;

37. Roadmap Epigenomics Mapping Consortium. Integrative analysis of 111 reference human epigenomes. Nature. 2015:518:317-30.

38. GTEx Consortium. Genetic effects on gene expression across human tissues. Nature. 2017:550:204-13,

39. Kapusta A, Kronenberg Z, Lynch VJ, Zhuo X, Ramsay L, Bourque G, Yandell $M$, Feschotte $C$. Transposable elements are major contributors to the origin, diversification, and regulation of vertebrate long noncoding RNAs. PLOS Genet. 2013;9:e1003470

40. Klaver B, Berkhout B. Comparison of $5^{\prime}$ and $3^{\prime}$ long terminal repeat promoter function in human immunodeficiency virus. J Virol. 1994; 68(6):3830-40.

41. Lavie L, Esther Maldener E, Brook Brouha B, Meese EU, Mayer J. The human L1 promoter: variable transcription initiation sites and a major impact of upstream flanking sequence on promoter activity. Genome Res. 2004;14:2253-60.

42. Walter M, Teissandier A, Pérez-Palacios R, Bourchis D. 2016. An epigenetic switch ensures transposon repression upon dynamic loss of DNA methylation in embryonic stem cells. elife. 2016;5 e11418

43. Daussy C, et al. T-bet and Eomes instruct the development of two distinct natural killer cell lineages in the liver and in the bone marrow. J Exp Med. 2014;3:563-77.

44. Gao F, et al. Integrated analyses of DNA methylation and hydroxymethylation reveal tumor suppressive roles of ECM1, ATF5, and EOMES in human hepatocellular carcinoma. Genome Biol. 2014;15:533-46. 
45. Guo P, Dong X-Y, Zhao KW, Sun X, Li Q, Dong J-T. Estrogen-induced interaction between KLF5 and estrogen receptor (ER) suppresses the function of ER in ER-positive breast cancer cells. Int J Cancer. 2010; 126(1):81-9.

46. Chen C, Bhalala HV, Qiao H, Dong JT. A possible tumor suppressor role of the KLF5 transcription factor in human breast cancer. Oncogene. 2002;21: 6567-72.

47. Nagaoka K, Fujii K, Zhang H, Usuda K, Watanabe G, Ivshina M, Richter JD. CPEB1 mediates epithelial-to-mesenchyme transition and breast cancer metastasis. Oncogene. 2016;35:2893-901.

48. The ENCODE Project Consortium. An integrated encyclopedia of DNA elements in the human genome. Nature. 2012;489:57-74.

49. Furuyama K, et al. 2010. Continuous cell supply from a Sox9-expressing progenitor zone in adult liver, exocrine pancreas and intestine. Nat Genet. 2010;43(1):35-42.

50. Cattoretti G, Angelin-Duclos C, Shaknovich R, Zhou H, Wang D, Alobeid B. PRDM1/Blimp-1 is expressed in human B-lymphocytes committed to the plasma cell lineage. J Pathol. 2005;206:76-86.

51. Cohen CJ, Lock WM, Mager DL. Endogenous retroviral LTRs as promoters for human genes: a critical assessment. Gene. 2009;448:105-14.

52. Janoušek V, Laukaitis CM, Yanchukov A, Karn R. The role of retrotransposons in gene family expansions in the human and mouse genomes. Genome Biol Evol. 2016;8:2632-50.

53. Saykally JN, Dogan S, Cleary MP, Sanders MM. The ZEB1 transcription factor is a novel repressor of adiposity in female mice. PlosONE. 2009;4(12):e8460.

54. Gubelmann C, et al. Identification of the transcription factor ZEB1 as a central component of the adipogenic gene regulatory network. elife. 2014;3:e03346.

55. Leow SC, et al. The transcription factor SOX6 contributes to the developmental origins of obesity by promoting adipogenesis. Development. 2016;143:950-61.

56. Alexandrov IM et al. 2012. Cytoplasmic Polyadenylation Element Binding Protein Deficiency Stimulates PTEN and Stat3 mRNA Translation and Induces Hepatic Insulin Resistance. Plos Genet. 2012;8(1):e1002457.

57. He G, Karin M. NF-KB and STAT3 - key players in liver in ammation and cancer. Cell Res. 2011;21:159-68.

58. Kelley D, Rinn J. Transposable elements reveal a stem cell-specific class of long noncoding RNAs. 2012:13(11):R107.

59. Beck CM, et al. LINE-1 Retrotransposition activity in human genomes. Cell. 2010;141:1159-70

60. Muotri AR, Chu VT, Marchetto MC, Deng W, Moran JV, Gage FH. Somatic mosaicism in neuronal precursor cells mediated by L1 retrotransposition. Nature. 2005;435(7044):903-10.

61. Coufal NG, Garcia-Perez JL, Peng GE, Yeo GW, Mu Y, Lovci MT, Morell M, O'Shea KS, Moran JV, Gage FH. L1 retrotransposition in human neural progenitor cells. Nature. 2009;460(7259):1127-31.

62. Upton KR, et al. Ubiquitous L1 mosaicism in hippocampal neurons. Cell. 2017;161:228-39.

63. Sur D, et al. Detection of the LINE-1 retrotransposon RNA-binding protein ORF1p in different anatomical regions of the human brain. Mob DNA. 2017;8:17.

64. Savage AL, et al. An evaluation of a SVA retrotransposon in the FUS promoter as a transcriptional regulator and its association to ALS. PLoS One. 2014;9(6):e90833.

65. Smit A, Hubley R, Green P. RepeatMasker open 4.0. 2013-2015. Http:// www. repeatmasker.org. Benjamini $Y$, Hochberg $Y$. Controlling the false discovery rate: a practical and powerful approach to multiple testing. J R Stat Soc B. 1995;57:289-300.

66. Quinlan AR, Hall IM. BEDTools: a flexible suite of utilities for comparing genomic features. Bioinformatics. 2010;26:841-2.

67. Benjamini Y, Hochberg Y. Controlling the false discovery rate: a prac- tical and powerful approach to multiple testing. J R Stat Soc B. 1995;57:289-300.

68. Bailey $T L$, et al. MEME SUITE: tools for motif discovery and searching. Nucleic Acids Res. 2009;37:W202-8.

69. R Core Team. $R$ : a language and environment for statistical computing. In: $R$ Foundation for Statistical Computing, Vienna, Austria. 2016. https://www.Rproject.org/.

70. Wickham H. ggplot2: elegant graphics for data analysis. 2009. SpringerVerlag, New York.

Ready to submit your research? Choose BMC and benefit from:

- fast, convenient online submission

- thorough peer review by experienced researchers in your field

- rapid publication on acceptance

- support for research data, including large and complex data types

- gold Open Access which fosters wider collaboration and increased citations

- maximum visibility for your research: over $100 \mathrm{M}$ website views per year

At BMC, research is always in progress.

Learn more biomedcentral.com/submissions 